\title{
Generalization of space-filling bearings to arbitrary loop size
}

\author{
G Oron $\dagger \ddagger$ and H J Herrmann $\dagger$ \\ $\dagger$ Laboratoire de Physique et Mécanique des Milieux Hétérogènes, UMR CNRS 7636, École de \\ Physique et de Chimie Industrielles de la ville de Paris, 10 rue Vauquelin, 75231 Paris Cedex 05, \\ France \\ E-mail: oron@pmmh.espci.fr
}

Received 7 June 1999

\begin{abstract}
A model for space-filling packing of discs rolling sliplessly on each other is presented This model is an extension of the model presented by Herrmann et al to a basic loop size of an arbitrary even number of discs. This model might have application in geophysics, dense granular flows and turbulence. We show the existence and uniqueness of such constructions, analytically, for loop size up to eight, and numerically for bigger basic loop sizes, and make precise the algorithm to construct them. A classification of the solution is also given and extensive studies of the fractal dimension are performed, showing that it varies considerably with the base loop size.
\end{abstract}

\section{Introduction}

Is it possible to fill a two-dimensional (2D) plane with gears all rolling on each other such that the entire area is covered with wheels? The study of such a question emerged in the context of tectonic plate movement in $[1,2]$ as a possible explanation of the enigmatic geological phenomena known as 'seismic gaps' [3,4]. Seismic gaps are regions along an active plate boundary where two tectonic plates can creep on each other without liberating, in the form of earthquakes or heat, the expected amount of energy from frictional forces. It can be conceived that the granular material filling the space between the two plates, known as 'fault gauge', is in fact partially arranged in rolls in such a manner that it behaves like gears in a bearing, rolling sliplessly on each other, and hence not exerting much frictional work, thus permitting a creep with a very low energetic cost. Other contexts where such constructions might play a role are turbulence (especially 2D) and dense granular flows.

Since any two circles in contact are rotating in opposite directions, a 2D packing of bearings has no rotational frustration if and only if any closed loop of touching circles in the packing has an even number of discs.

Tiling the space with circles by iteratively placing, between every three circles, a circle tangentially touching all three is an old problem known as 'Apollonian packing' and dates back to the Greek mathematician Apollonius of Perga (now known as Murtana in Antalya, Turkey) who lived around $200 \mathrm{BC}$ and was the first to show how to construct a circle which is at a tangent to three given circles. In modern times, much work has been done mainly aimed at obtaining estimates of the fractal dimension of Apollonian packings, which is about 1.306, by both theoretical estimations [5,6] and numerical calculations [7]. (In this paper we do not

† Author to whom correspondence should be addressed at Departament de Física Fonamental, Universitat de Barcelona, Av. Diagonal 647, 08028 Barcelona, Spain. 
distinguish between the 'Hausdorff dimension' and the 'box-counting dimension' and suppose them equal. For further discussion of this issue see [8].) This packing presents exactly the opposite characteristic of what we are looking for, since every closed loop has an odd number of bearings, hence the Apollonian packing is completely 'locked'.

The existence of strip filling with discs without rotational frustration, baptized 'spacefilling bearings' (SFBs), was proven in [1] using a method based on iteratively applying conformal transformations: reflections, translations and inversions. It was shown that there are two different families, denoted by families 1 and 2 ( $F 1$ and F2, respectively), each one having different realizations parametrized by two positive integers $\left(n_{1}, n_{2}\right)$. The fractal dimension of these families was also numerically estimated to lie in the range between 1.306 and 1.520 [1,7]. This idealized gauge modelling permitted a theoretical estimate of properties such as faultgauge size distributions as measured in [9-11], and remanent magnetization in rocks issued from cataclastic shear flow [12].

Nevertheless, the study in [1] was limited to SFBs with a 'basic loop' size of four elements: i.e., constructions in which all closed loops of the bearings are, at least, four discs in size (this term will be defined more precisely below).

The aim of this work is to prove the existence and study the properties of SFBs with a basic loop size larger than four discs. We will first introduce a variant of the study presented in [1] which is more adequate for the general case and that will lead us to conclude the existence of SFBs with a larger, even number of bearings in their basic loop. We will show that, as for the four-fold loop case, for each given basic loop size, there exists two topologically different families of SFBs, each one having an infinite number of different realizations described by two integers. This will be followed by a study of the properties for the different possible solutions, especially their fractal dimension.

The layout of this paper is as follows: in section 2 we show the method used for the construction of general loop size SFBs. In particular, we discuss the working assumptions (section 2.1), the notation used (section 2.2), the choice of the transformations (section 2.3), the conditions that the transformations must fulfil (see section 2.4 with complementary mathematical details given in the appendix) and how the construction algorithm is effectively implemented utilizing a computer (section 2.5). In section 3 we discuss the issue of the fractality of the constructed set, and in section 4 we present some examples of general loop size SFBs we have calculated and study their size distribution. Section 5 contains a discussion of the results in the light of the experimental work presented in [9-11,13].

A more detailed version of the work presented here can be found in [14] in which the working assumptions and the choice of the transformations (sections 2.1 and 2.3, respectively) are discussed extensively.

\section{Construction of a generalized base loop size SFB}

\subsection{Working assumptions}

In the construction of SFBs we fix several working assumptions. First of all we use homographic transformations (also known as Möbius maps) of the first (that conserve the orientation) and of the second kind (that do not conserve it). In the complex representation of the plane the homographic transformations of the first kind can be written as

$$
\mathcal{C}(z)=\frac{a z+b}{c z+d} \quad \text { with } \quad a, b, c, d \in \mathbb{C}
$$


or represented in matrix form as

$$
\mathcal{M}=\left(\begin{array}{ll}
a & b \\
c & d
\end{array}\right)
$$

with $\operatorname{det}(\mathcal{M})=a d-b c=1$. In particular, these transformations contain the rotations by an angle $\theta(a=\exp (\mathrm{i} \theta / 2), d=\exp (-\mathrm{i} \theta / 2), b=c=0)$, translations of a vector $v \in \mathbb{C}$ ( $a=d=1, b=v, c=0)$ and dilatations by a factor $\alpha\left(a=\alpha^{\frac{1}{2}}, b=c=0, d=\alpha^{-\frac{1}{2}}\right)$. The homographic transformations of the second kind are defined in a manner similar to (1), but replacing $z$ by $\bar{z}$, the conjugate of $z$. The last category contains, in particular, the inversions around a circle $\left(a=\mathrm{i} v / r, b=\mathrm{i}\left(r^{2}-v^{2}\right) / r, c=\mathrm{i} / r\right.$ and $d=-\mathrm{i} \bar{v} / r$, where $r$ is the radius of the circle and $v$ is its centre) and reflection about a straight line (if this line intersects the real axis in $u$ making an angle $\phi$ with it, then $a=\exp [\mathrm{i} \phi], b=-2 u \mathrm{i} \sin (\phi), c=0$ and $d=\exp [-\mathrm{i} \phi])$. Recall that the homographic transformations of the first kind form a group for the composition (given by the product in the matrix representation), while those of the second kind do not. Moreover, the composition of two homographic transformations of the second kind is a transformation of the first kind, while the composition of two transformations of different kinds gives a transformation of the second kind [15].

The choice of the homographic transformations resides in the fact that they transform a circle into a circle (a straight line is considered to be a circle with infinite radius) and conserve the topology; if the source was a closed loop of an even number of discs then an image produced by any of the above transformations will be a loop of the same number of discs. Hence, if we start with a 'seed' of any even number of elements and apply any sequence of these transformations, and if the obtained set of circles is a packing, i.e. if no overlap occurs, then the ensemble of images obtained will all have the same topology and hence the SFB condition will be satisfied. The number of circles in the seed is called the 'base loop size' (BLS). Since every one of the circles is an image of another produced by one of the transformations, the final set must be invariant under any of the constructing transformations.

The second working assumption is that, for simplicity in the calculations and in the light of the geometry of the physical system, we choose to work in a strip geometry of unit width, or in other words, we choose the seed to be composed of two parallel lines and a sequence of circles connecting them. Since the two straight lines 'touch' at the point of infinity the seed is indeed a closed loop. This property will be used later in order to determine the parameters of the transformations.

This choice of geometry does not limit the generality of the results since once the construction of the strip is complete one can apply any of the transformations cited above, or any of their combinations in order to get different geometries, such as a disc, as shown in figure 1. One can even combine different realizations of SFBs, for example, putting different strip configurations on top of one another, or replacing one of the discs in the set with a whole SFB in a disc geometry, etc. Note also that, stricto sensu, simple shearing is not possible with the SFB model since the parallel lines must move in the same direction. It is, however, possible when allowing a slight curvature of the boundaries as may be obtained near a contact point of two circles. Since an interface is never perfectly straight, this artifact has no physical importance. One last assumption is that the strip has a translational invariance with a period of $2 a$. Since $a$ is now any number this is not a restriction.

\subsection{Notation}

Before continuing any further, let us define some of the notation we use in what follows (see figures 2 and 3 for an illustration of this notation). 


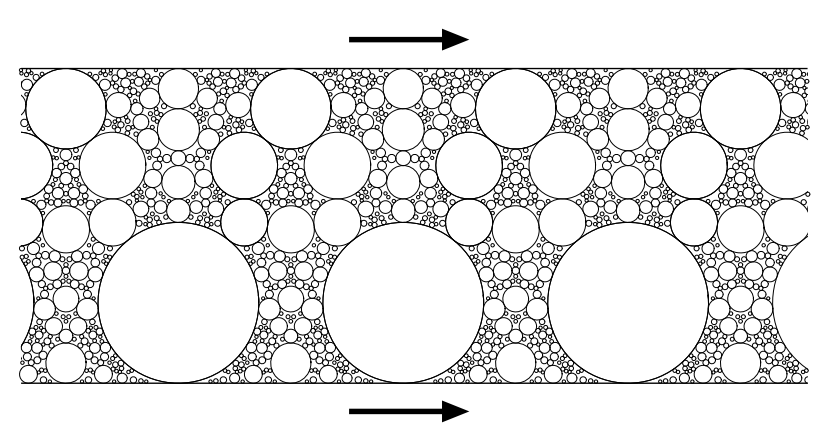

(a)

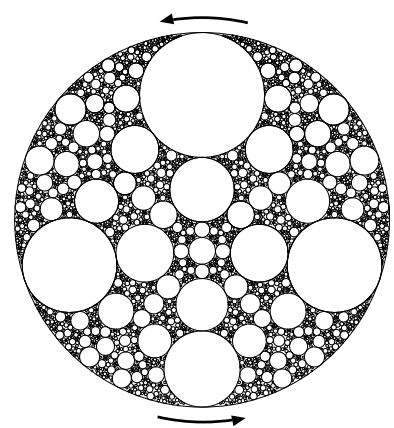

(b)

Figure 1. Rolling SFBs of F1 with a BLS of six elements, $n_{1}=0$ and $n_{2}=1$ (the meaning of these parameters is explained in the text). (a) A strip geometry and (b) a circular geometry obtained from $(a)$ by an inversion.

(i) We denote the BLS as $l$, or the size of the seed as discussed in section 2.1. We also define $p=l / 2-1$.

(ii) $\Gamma_{i}$, where $i=0, \ldots, l-1$, will refer to the $i$ th circle in the seed, where $\Gamma_{0}$ is the lowest one (the lower line) and $\Gamma_{l-1}$ is the uppermost one (the upper line).

(iii) $A_{i}$, where $i=0, \ldots, l-2$, are the contact points $\Gamma_{i} \cap \Gamma_{i+1}$.

(iv) Finally, $d_{i}$, where $i=1, \ldots, l-2$ designates the distance $\overline{A_{0} A_{i}}$.

\subsection{Choice of the transformations}

In order to cover the plane in the most effective way we will choose the biggest class of homographic transformations compatible with the hypothesis. Let us first focus our attention on the inversions. It is clear that if the strip is to be invariant under them, these inversions must be centred on a contact point between two circles. This is because in this case the image of these two circles would be two parallel lines. Since any circle is either in the seed or is an image of a circle which is in it, we can restrict our choice to the contact point in the seed $A_{i}$. First, we arbitrarily choose point $A_{0}$ to be the centre of the first inversion $\mathcal{I}_{1}$ (see figures 2 and 3). Since the seed must be invariant under this inversion it must be arranged so that $A_{0}$, $A_{i}$ and $A_{i^{\prime}}=\mathcal{I}_{1}\left(A_{i}\right)$ are aligned. One can show that this also implies that all of the contact points in the seed $A_{i}$ are aligned along $\overline{A_{0} A_{l-2}}$ [14].

Two different cases are possible, either $\overline{A_{0} A_{l-2}} \perp \Gamma_{0}$, or otherwise. In the first case we will deal with 'F2' construction (shown in figure 3) and in the latter case with 'F1' (see figure 2).

When reflections are considered for the two families the strip geometry and the translational invariance $\mathcal{T}=z \rightarrow z+2 a$ permit only reflection symmetry about the vertical lines $s_{1}$ and $s_{2}$ of equations $\operatorname{Re}(z)=0$ and $\operatorname{Re}(z)=a$ respectively, shown in figures 2 and 3 . We denote these reflections $\mathcal{R}_{1}$ and $\mathcal{R}_{2}$ respectively. In the F1 case this implies that consecutive copies of the seed touch each other.

It is easy to see that these transformations alone do not fill the whole strip but only the region close to $A_{0}$, hence we must add another inversion-we choose an inversion $\mathcal{I}_{2}$ centred at $A_{l-2}$ and of radius $r_{2}$. One can check, by trying to solve the equation systems that we present in the following (equations (4) and (5)), that this is the only topological situation possible for 


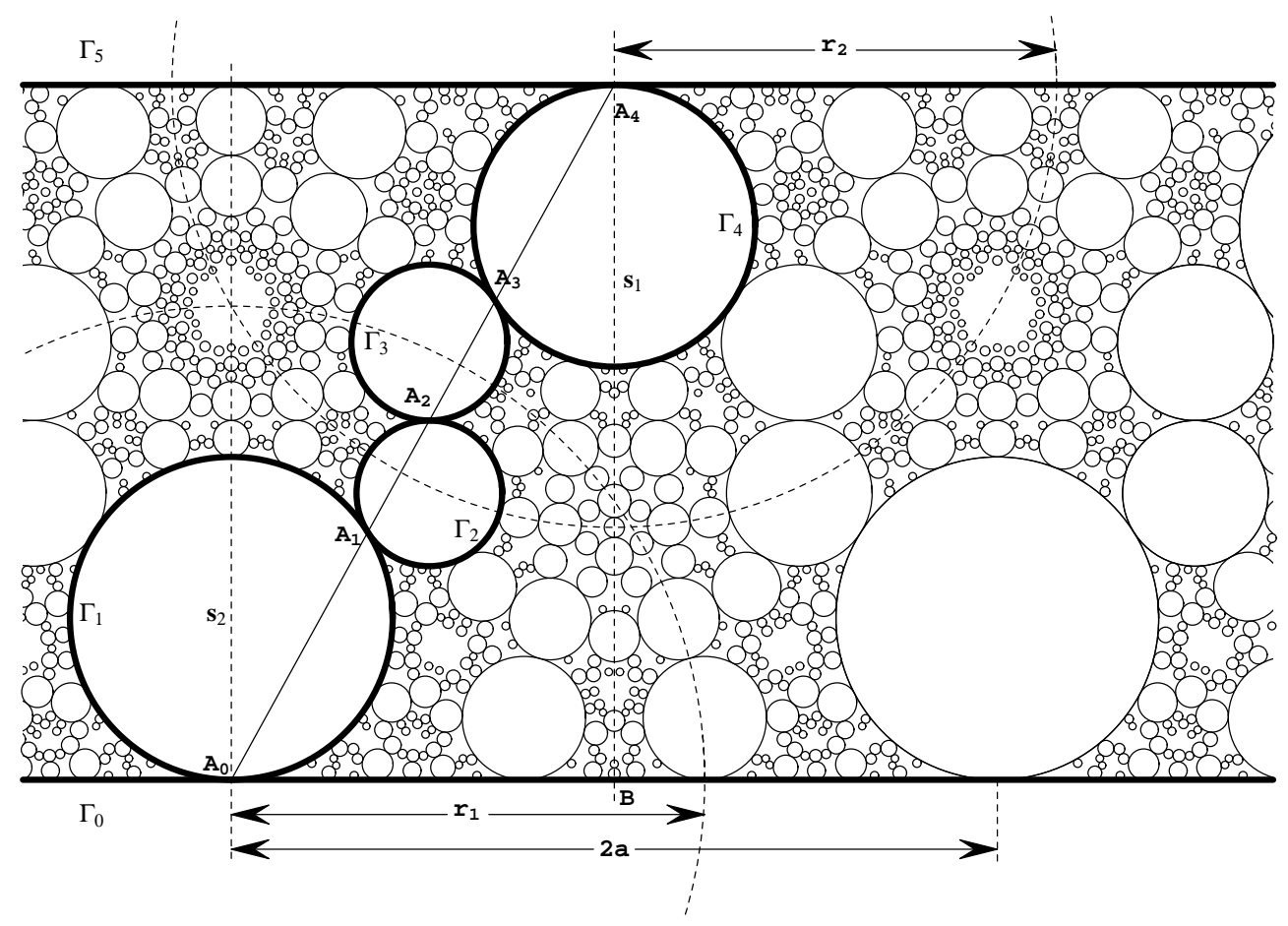

Figure 2. Notation used in the construction of F1 strips. The strip shown depicts six-element BLS SFBs with $n_{1}=2$ (for the inversion centred at $A_{0}$ ) and $n_{2}=3$ (inversion centred at $A_{5}$ ). In this figure we only kept circles with radii greater than $10^{-2}$ of the strip width. The dashed circles are the inversion circles for $\mathcal{I}_{1}$ and $\mathcal{I}_{2}$, of radii $r_{1}$ and $r_{2}$, respectively. The lines $s_{1}$ and $s_{2}$ are the axes of the reflections $\mathcal{R}_{1}$ and $\mathcal{R}_{2}$, respectively. The circles and lines drawn in bold are elements of the seed. The notation used for the seed circles $\Gamma_{0}, \ldots, \Gamma_{5}$, the contact points $A_{0}, \ldots, A_{4}$, and the spatial periodicity $2 a$, are also displayed.

the inversions, i.e. centred at contact points separated by a distance of two circles from one another.

The question one should answer in order to complete the construction of the SFB is 'what are the different parameters (radii of the inversions $r_{1}$ and $r_{2}, a$ ) that will create a packing?' In other words, no overlap of circles occurs after each transformation.

It was shown in [1], using the packing properties proved in [16] which make use of reflection groups, that in the case of SFBs the transformations introduced above produce a packing if and only if there exists a base in which $\mathcal{R} \mathcal{I}$ is a translation or a rotation of an angle $2 \pi / k, k \in \mathbb{N}^{+}$. This condition is equivalent to (see [14] for more details)

$$
\begin{aligned}
r_{1}^{2} & =a^{2} z_{n_{1}} \\
r_{2}^{2} & =a^{2} z_{n_{2}}
\end{aligned}
$$

where

$$
z_{k} \equiv\left(\cos \frac{\pi}{k+3}\right)^{-2} \quad k \in \mathbb{N} .
$$

$n_{1}+3$ and $n_{2}+3$ might be interpreted as the size of the group of transformations that can be created by the composition of the respective $\mathcal{R} \mathcal{I}$ with themselves, i.e. $\left\{(\mathcal{R} \mathcal{I})^{(n)}, n \in \mathbb{N}\right\}$. Or, in other words, $n_{1}+2$ and $n_{2}+2$ are the number of transformations $\mathcal{R} \mathcal{I}$ that one should apply to 


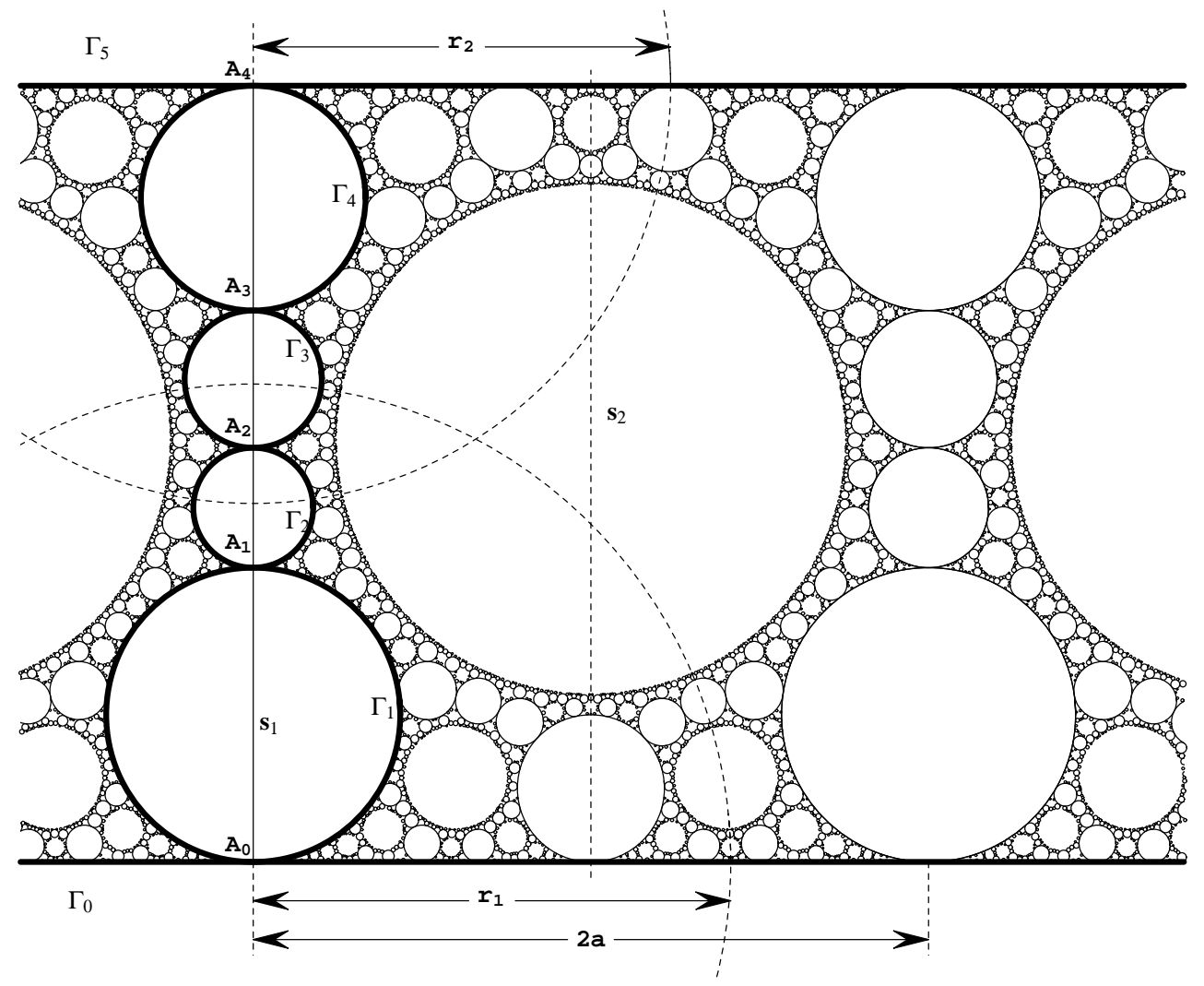

Figure 3. Notation used in the construction of F2 strips. The strip shown depicts six-element BLS SFBs with $n_{1}=1$ (for the inversion centred at $A_{0}$ ) and $n_{2}=2$ (inversion centred at $A_{5}$ ). In this figure we only kept circles with radii greater than $10^{-3}$ of the strip width. The dashed circles are the circles of the inversions $\mathcal{I}_{1}$ and $\mathcal{I}_{2}$, of radii $r_{1}$ and $r_{2}$, respectively. The lines $s_{1}$ and $s_{2}$ are the axes of the reflections $\mathcal{R}_{1}$ and $\mathcal{R}_{2}$, respectively. The circles and lines drawn in bold are elements of the seed. The notation used for the seed circles $\Gamma_{0}, \ldots, \Gamma_{5}$, the contact points $A_{0}, \ldots, A_{4}$, and the spatial periodicity $2 a$, are also displayed.

the seed before no new images might be created. This fact will be used later in the construction itself (see section 2.5).

\subsection{The equation systems for the seed}

In this section we present the equations that need to be solved in order for us to get the parameters of the constructing transformations. In both cases, F1 and F2, we will write the invariance of the seed under the inversions followed by the packing condition given in (3). Since any circle is an image of the seed these condition suffice to ensure that the whole construction adheres to the SFB conditions, i.e., that it forms a packing and that the no-slip rolling condition is satisfied everywhere.

2.4.1. F1. The invariance condition of the seed under the inversion $\mathcal{I}_{1}$ centred at $A_{0}$, and of radius $r_{1}$, gives

$$
d_{1} d_{2 p}=r_{1}^{2} \quad d_{2} d_{2 p-1}=r_{1}^{2}, \ldots, d_{p} d_{p+1}=r_{1}^{2} .
$$


The invariance condition of the same seed under the inversion $\mathcal{I}_{2}$ centred at $A_{l-2}=A_{2 p}$, and of radius $r_{2}$, gives

$$
\begin{aligned}
& d_{2 p}\left(d_{2 p}-d_{2 p-1}\right)=r_{2}^{2} \\
& \left(d_{2 p}-d_{1}\right)\left(d_{2 p}-d_{2 p-2}\right)=r_{2}^{2}, \ldots,\left(d_{2 p}-d_{p-1}\right)\left(d_{2 p}-d_{p}\right)=r_{2}^{2} .
\end{aligned}
$$

We apply Pythagoras' theorem to the triangle $A_{0} A_{l-2} B$ :

$$
a^{2}+1=d_{2 p}^{2}
$$

and the packing condition as discussed above (equation (3)):

$$
r_{1}^{2}=a^{2} z_{n_{1}} \quad r_{2}^{2}=a^{2} z_{n_{2}} \quad z_{k} \equiv\left(\cos \frac{\pi}{k+3}\right)^{-2} \quad k \in \mathbb{N}
$$

to obtain $l+1$ nonlinear equations with $l+1$ unknowns.

The nonlinear equation system obtained from $(4 a)-(4 d)$ can be solved analytically for small $l$ (at most eight, see appendix A.2), and numerically for larger $l$, yielding multiple solutions. However, in all studied cases we have found only one positive and ordered solution, i.e. so that $0<d_{1}<d_{2}<\cdots<d_{l-2}$. This solution provides us with $r_{1}, r_{2}$ and $a$, hence permitting the iterative construction of the SFB strip.

2.4.2. F2. The equations describing the invariance of the seed under the inversions $\mathcal{I}_{1}$ and $\mathcal{I}_{2}$ are the same as those obtained for F1 in (4), but instead of (4c) we now have $d_{2 p}=1$, which follows from the orthogonality of $\overline{A_{0} A_{2 p}}$ with $\Gamma_{0}$ and $\Gamma_{l-1}$, and from the fact that the strip width is unity.

The condition to obtain a packing is the same as for F1 (equation (3)), thus the nonlinear equation system to solve in the $\mathrm{F} 2$ case is

$$
\begin{aligned}
& r_{1}^{2}=d_{1} d_{2 p} \quad r_{1}^{2}=d_{2} d_{2 p-1}, \ldots, r_{1}^{2}=d_{p} d_{p+1} \\
& r_{2}^{2}=d_{2 p}\left(d_{2 p}-d_{2 p-1}\right), \ldots, r_{2}^{2}=\left(d_{2 p}-d_{p-1}\right)\left(d_{2 p}-d_{p}\right) \\
& 1=d_{2 p} \\
& r_{1}^{2}=a^{2} z_{n_{1}} \\
& r_{2}^{2}=a^{2} z_{n_{2}} \quad \text { where } \\
& z_{k} \equiv\left(\cos \frac{\pi}{k+3}\right)^{-2} \quad k \in \mathbb{N} .
\end{aligned}
$$

This equation system is slightly easier to solve than the equation for the F1 case and was solved analytically for small $l$ (at most eight, see appendix A. 3 for further details) and numerically for larger $l$. As for F1, multiple solutions were found but only one of these solutions was positive and ordered. The solution provides us with $r_{1}, r_{2}$ and $a$, hence permitting the iterative construction of the SFB strip.

\subsection{The implementation of the construction algorithm}

The construction algorithm is based on the fact that translations of circles far from the inversion points will, when inversion is applied, create circles with smaller and smaller radii that will fill the empty wedges near the seed. Given $l, n_{1}, n_{2}$ and the family, we first write the corresponding equation system (equations (4) or (5)), solve it analytically, for small $l$ (see appendices A.2 and A.3 for further details) or numerically for bigger $l$. For $l$ smaller than 20, Mathematica's implementation of Jenkins-Traub algorithm for polynomial roots calculation was used. This method provides all of the roots from which the ordered, all positive solution was picked. For 
higher $l$ the damped Newton method implemented in Mathematica was used. In all cases, 16 decimal precision floating point numbers were used. For further details on the implementation of these algorithms see [17].

When $r_{1}, r_{2}$ and $a$ are obtained, the strip is initialized by placing $\Gamma_{0}$ and $\Gamma_{l-1}$ (there is no need to add the other elements of the seed since they will all be created as images of $\Gamma_{0}$ and $\left.\Gamma_{l-1}\right)$. The sequence of transformations

$$
\mathcal{R}_{1} \mathcal{I}_{1} \quad\left(\mathcal{R}_{1} \mathcal{I}_{1}\right)^{(2)}, \ldots,\left(\mathcal{R}_{1} \mathcal{I}_{1}\right)^{\left(n_{1}+2\right)}
$$

is then applied. The same is done for the upper part of the strip with $\mathcal{R}_{2}, \mathcal{I}_{2}$ and $n_{2}$, followed by the translation $\mathcal{T}$. Only the circles with radii larger than a certain fixed size $\varepsilon$ are kept (most of the time we chose $\varepsilon=3 \times 10^{-4}$ and get up to about $4 \times 10^{4}$ circles, which gives enough statistics for the determination of the fractal dimension with reasonable calculation time). The process is repeated until no new circles can be created. An illustration of the construction procedure showing two iterations is given in figures 4 and 5 .

A technical difficulty resides in the fact that this procedure creates many repeated circles. These must be eliminated to have the correct statistics and in order to reduce computer memory consumption. The construction procedure was programmed using Mathematica symbolic calculation software in order to have control over the accuracy of the calculations. External $\mathrm{C}$ functions were used for the elimination of repeated circles which proved to be very time consuming, but with no risk of accuracy loss. The calculation time for $\varepsilon=3 \times 10^{-4}$ varies from several hours up to a few days (larger $n_{1}$ and $n_{2}$ ) on a $400 \mathrm{MHz}$ Pentium II computer.

\section{Fractality and fractal dimension}

The packings we have just constructed are fractal since the construction procedure is scale invariant (even though not in a simple manner) and has a fine, irregular structure [8]. To define the fractal dimension of these ensembles, one can consider the number $N(\varepsilon)$ of circles of radii larger than $\varepsilon$, the sum of their perimeters $s(\varepsilon)$ and the area not covered by the circles (or the 'porosity') $p(\varepsilon)$. If one denotes as $n(r)$ the distribution of the radii size per unit area, these quantities are linked by

$$
\begin{aligned}
& N(\varepsilon)=\int_{\varepsilon}^{\infty} n(r) \mathrm{d} r \\
& s(\varepsilon)=2 \pi \int_{\varepsilon}^{\infty} r n(r) \mathrm{d} r \\
& p(\varepsilon)=1-\pi \int_{\varepsilon}^{\infty} r^{2} n(r) \mathrm{d} r .
\end{aligned}
$$

If $n(r)$ obeys a simple power law $n(r) \sim r^{-\tau}$, then

$$
N(\varepsilon) \sim \varepsilon^{-d_{f}} \quad s(\varepsilon) \sim \varepsilon^{1-d_{f}} \quad p(\varepsilon) \sim \varepsilon^{2-d_{f}}
$$

where $d_{f}=\tau-1$ is the fractal dimension [18]. Because of these simple relations only the cumulative size distribution $N(\varepsilon)$, will be considered in the following.

\section{Examples of SFBs}

Let us now present the properties of different realizations of SFBs (different families, $l, n_{1}$ and $n_{2}$ ) produced using the construction method described in section 2.5. 


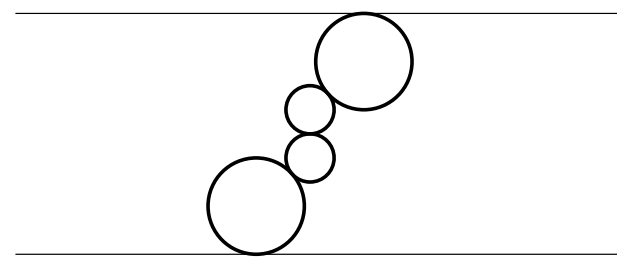

(i): the seed

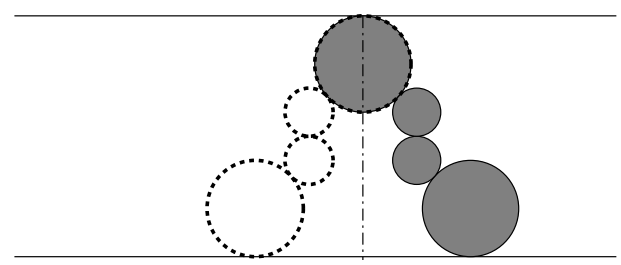

(iii): (i) after $\mathcal{R}_{1} \mathcal{I}_{1}$

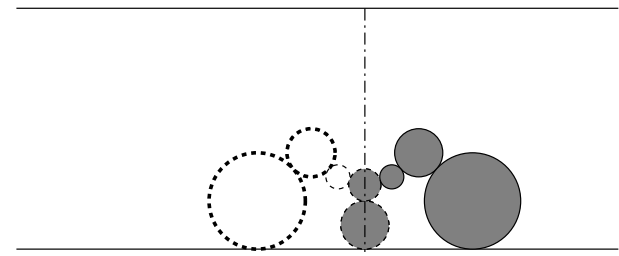

(v): (i) after $\left(\mathcal{R}_{1} \mathcal{I}_{1}\right)^{(2)}$

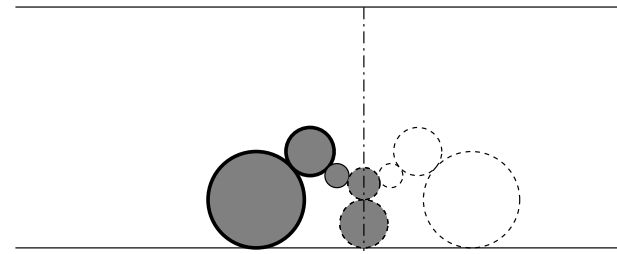

(vii): (i) after $\left(\mathcal{R}_{1} \mathcal{I}_{1}\right)^{(3)}$, no new circles may be produced

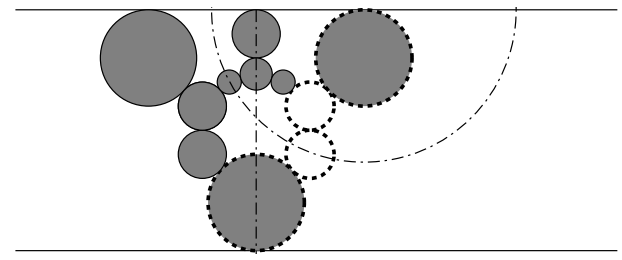

(ix): steps (ii) to (viii) applied for the upper part of the strip

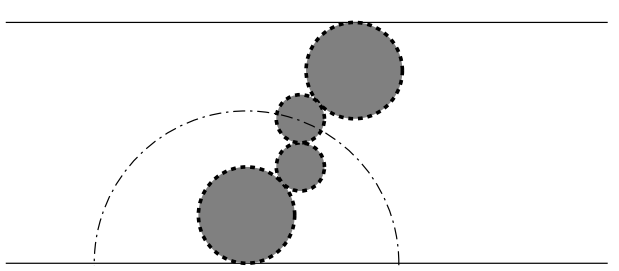

(ii): (i) after $\mathcal{I}_{1}$ - invariant

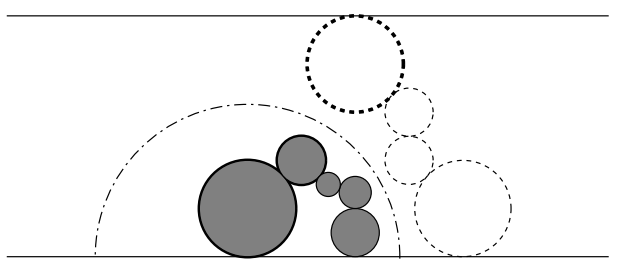

(iv): (i) after $\mathcal{I}_{1} \mathcal{R}_{1} \mathcal{I}_{1}$

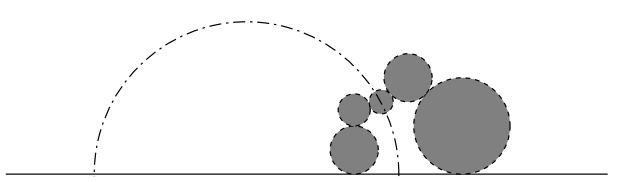

(vi): (v) is invariant by $\mathcal{I}_{1}$

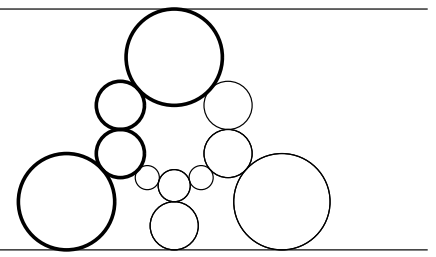

(viii): the set obtained, (i) $\cup($ iii $) \cup(v) \cup($ vii $)$

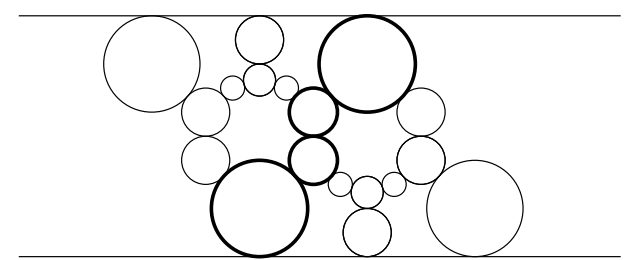

$(\mathrm{x})$ : (viii) $\cup(\mathrm{ix})$

Figure 4. Illustration of the SFB construction steps. The parameters used here are $l=6, n_{1}=1$ and $n_{2}=1$. We draw with a bold curve the elements of the seed, with dash-dotted lines and curves the transformation applied in the current step with a dashed curve the circles subject to the homographic transformation with their images shaded in grey. The sequence is continued in figure 5 .

In figure 6 we show examples of F1 SFB strips with different $l, n_{1}$ and $n_{2}$, while in figure 7 we present F2 strips with the same parameters. Figure 8 shows the evolution of a SFB with a BLS of six elements when $n_{1}$ and $n_{2}$ are varied. Notice that in many cases internal structures 


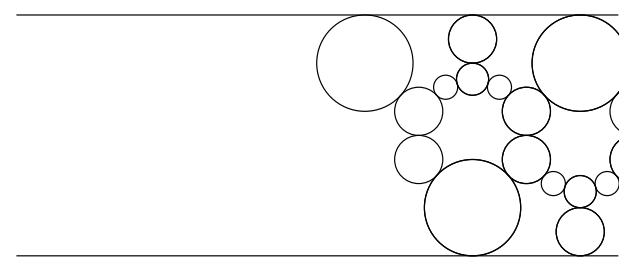

(xi): (x) translated by $2 a$, it will serve as source for the second iteration

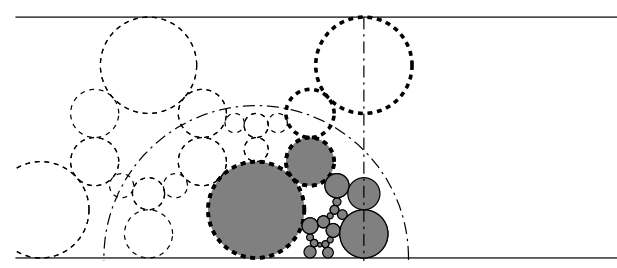

(xiii): (xi) after $\left(\mathcal{R}_{1} \mathcal{I}_{1}\right)^{(2)}$

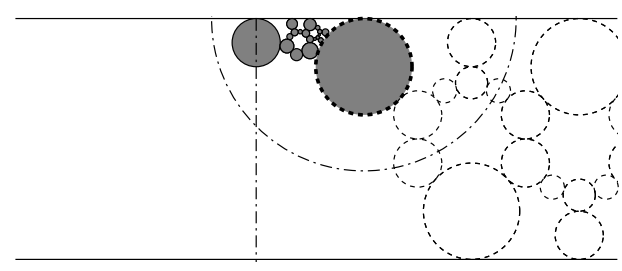

(xv): (xi) after $\mathcal{R}_{2} \mathcal{I}_{2}$

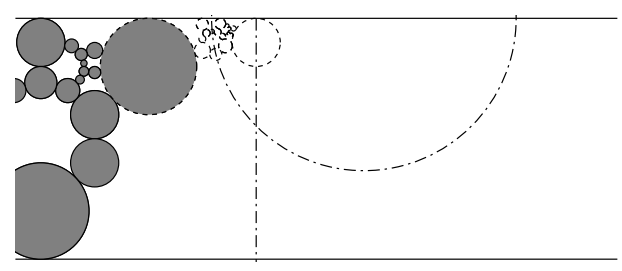

(xvii): (xi) after $\left(\mathcal{R}_{2} \mathcal{I}_{2}\right)^{(3)}$

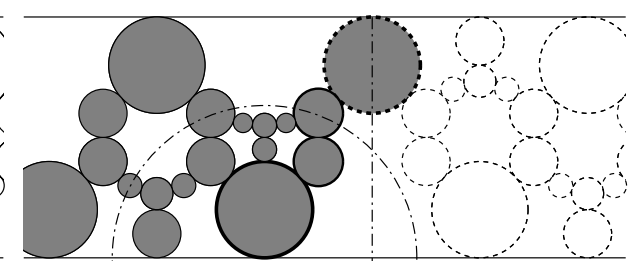

(xii): we re-apply steps (ii)-(xi) on (xi), after $\mathcal{R}_{1} \mathcal{I}_{1}$ we get

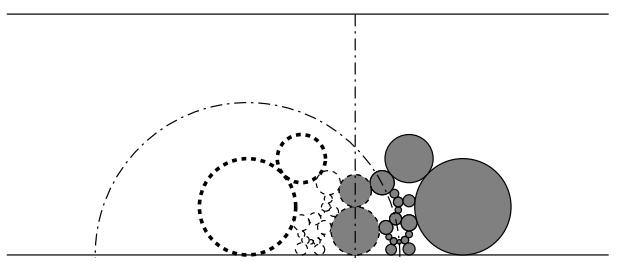

(xiv): (xi) after $\left(\mathcal{R}_{1} \mathcal{I}_{1}\right)^{(3)}$

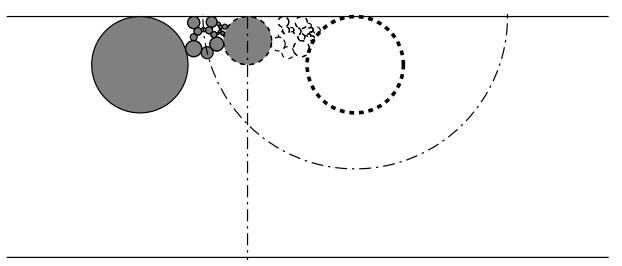

(xvi): (xi) after $\left(\mathcal{R}_{2} \mathcal{I}_{2}\right)^{(2)}$

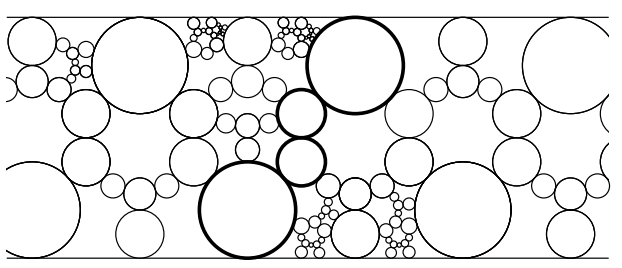

(xviii): the strip obtained after 2 iterations

Figure 5. Second part of the illustration of the SFB construction steps (see figure 4 for the first part).

such as lines or circles appear, where circles of small size accumulate. Notice, also, that for high BLS, circular 'holes' (and all their images) appear for both families. It is interesting to observe that these holes sometimes take the place of 'real' circles. For example, 4, (0, 0) of F2 in figure 7 , which has no holes, is topologically identical to the $F 1,6,(\infty, \infty)$ in figure 8 , with the only difference being that the top seed circle (and its images) in the F2 case are replaced by circular holes in the F1 case.

An example of the cumulative size distribution $N(\varepsilon)$ for two different realizations is shown in figure 9 . These distributions are clearly power laws and provide us with $d_{f}$ using (8). The 

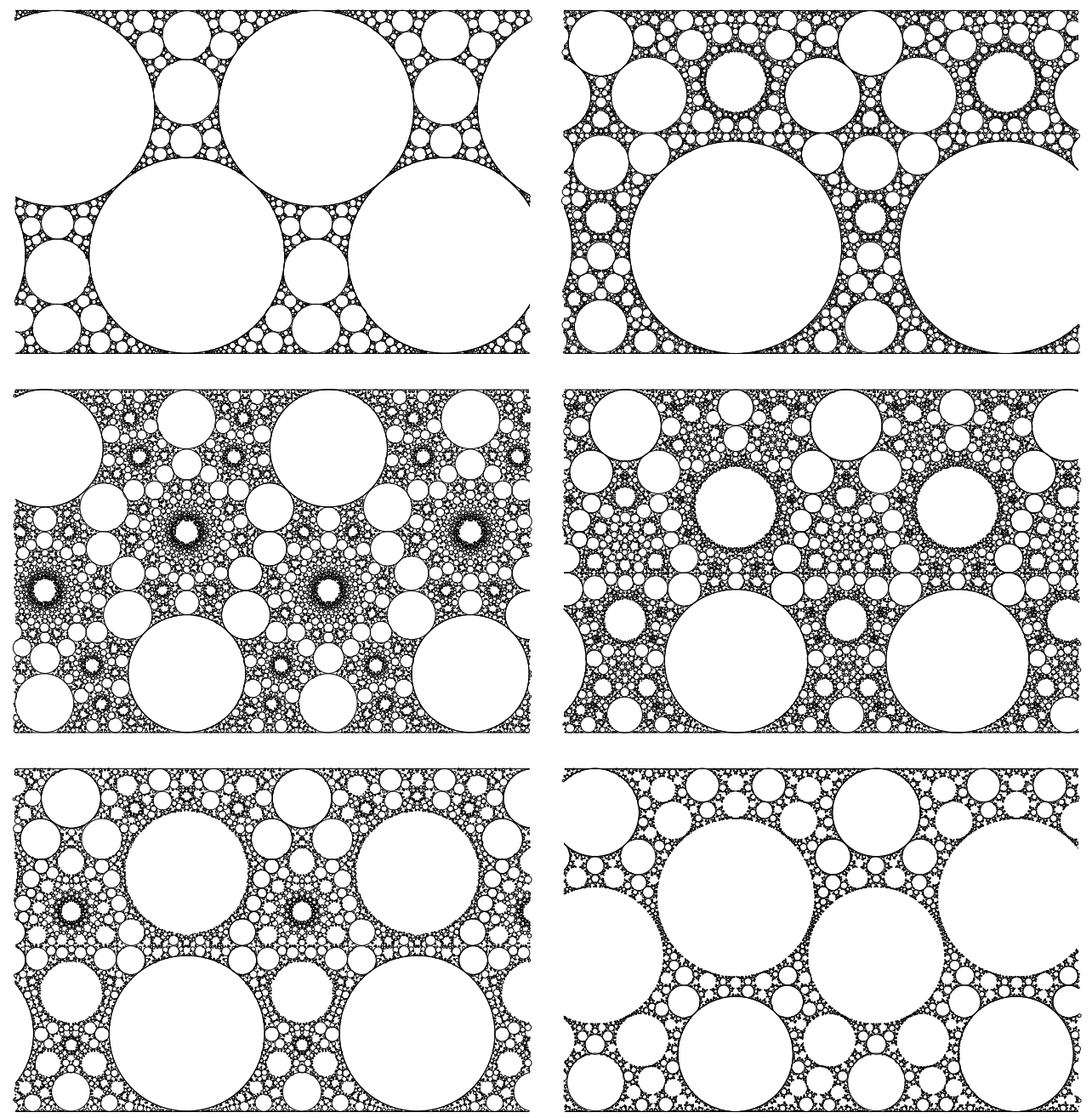

Figure 6. Some examples of F1 SFBs, for different $\left(n_{1}, n_{2}\right)$ and BLSs. Ordered from left to right and from top to bottom, in the format $l,\left(n_{1}, n_{2}\right): 4,(0,0), 6,(0,4), 8,(1,1), 10,(0,1), 12,(0,2)$ and $30,(1,2)$.

distribution deviates from the power law due to finite-size effects at radii greater than about 0.01 of the strip width.

Figure 10 gives some examples of the evolution of $d_{f}$ with $l$ for several different $\left(n_{1}, n_{2}\right)$. It shows that the evolution of $d_{f}$ is different for the two families. First of all, $d_{f}$ for F1 is nearly always larger than for F2 (F1 ranges from 1.304 to 1.803, and F2 ranges between 1.362 and 1.701), thus showing that the topological difference between the two families gives rise to large differences in the way the strip is filled. The behaviour for increasing $l$ is also different; while F2 is nearly always decreasing, F1 is increasing for low $l$ up to a maximum at $l=12$ and then decreases. Both families seem to converge to an asymptotic value for large $l$. Some fluctuations are observed for high $n_{1}$ or $n_{2}$. Another interesting point is that for $l=4$, $\left(n_{1}, n_{2}\right)=(\infty, \infty)$ the Apollonian packing is obtained, showing that it is a special case of SFB. The fractal dimension obtained in this case is $d_{f}=1.3044 \pm 0.009$ which is compatible 

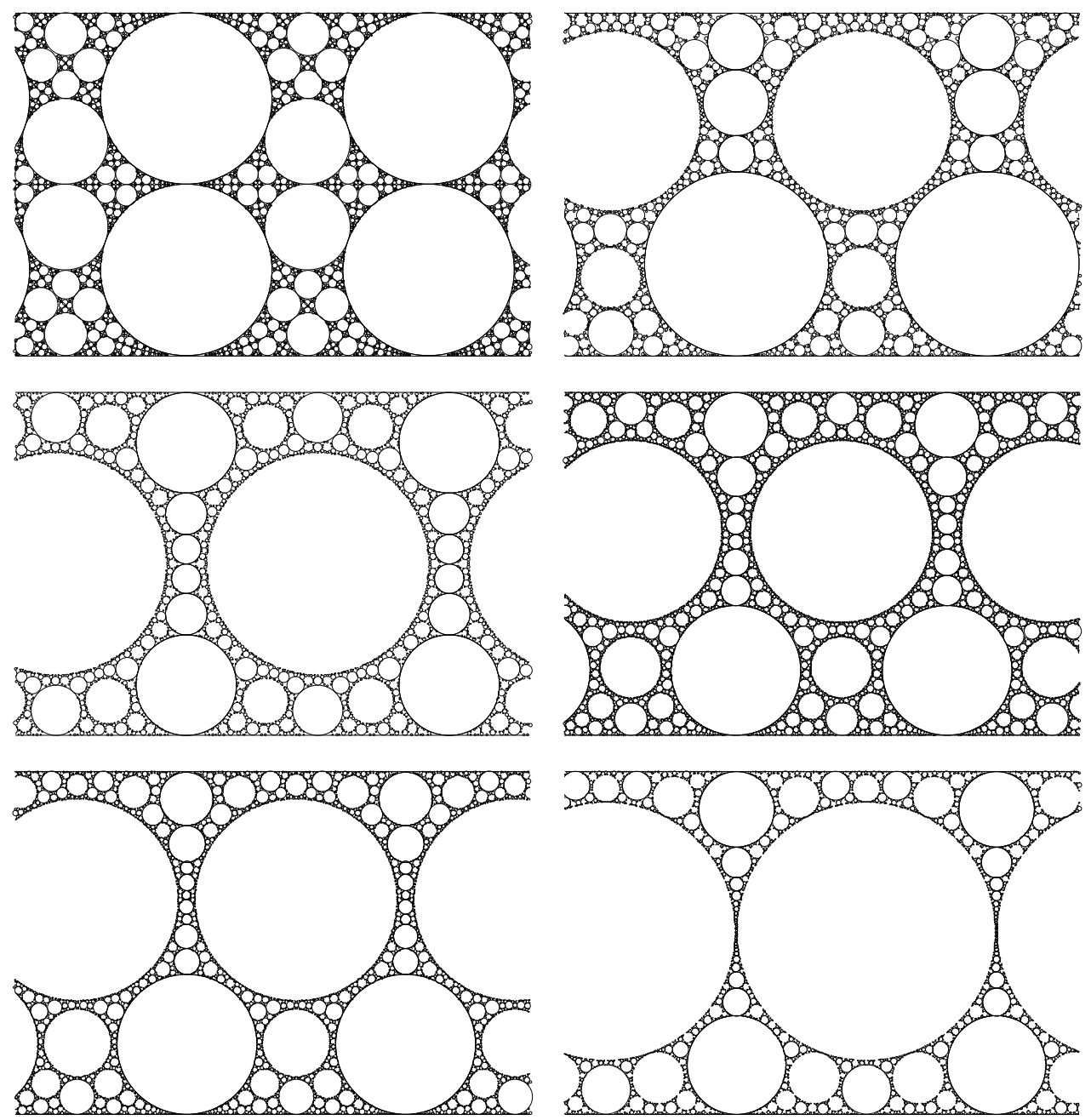

Figure 7. Some examples of F2 SFBs, for different $\left(n_{1}, n_{2}\right)$ and BLSs (the same as shown in figure 6). Ordered from left to right and from top to bottom, in the format $l,\left(n_{1}, n_{2}\right): 4,(0,0), 6$, $(0,4), 8,(1,1), 10,(0,1), 12,(0,2)$ and $30,(1,2)$.

with other values in the literature [6,7]. The value of the fractal dimension in this case is also the smallest among all SFBs calculated. This fact is compatible with a conjecture of Besicovitch which claims that the fractal dimension of the Apollonian packing is the smallest of all possible fillings of the plane $[19,20]$.

It is also interesting to study the part of the strip that is uncovered by the discs due to the existence of holes. From equation $(7 c)$ it is clear that this part is given by $p(0)$ and can be easily deduced from the power-law fit of $N(\varepsilon)$. The evolution of $p(0)$ with $l$ for different SFB realizations (the same as those of figure 10) is presented in figure 11. We find that, as for $d_{f}$, the two families evolve in a different range of $p(0)$ values, the second family having much higher values and hence being much emptier than the first family. On the other hand, the evolution of $p(0)$ is similar for the two families since $p(0)$ is always increasing. One other observation is that very few SFB realizations effectively fill the whole strip, i.e. cases where $p(0)=0$. 

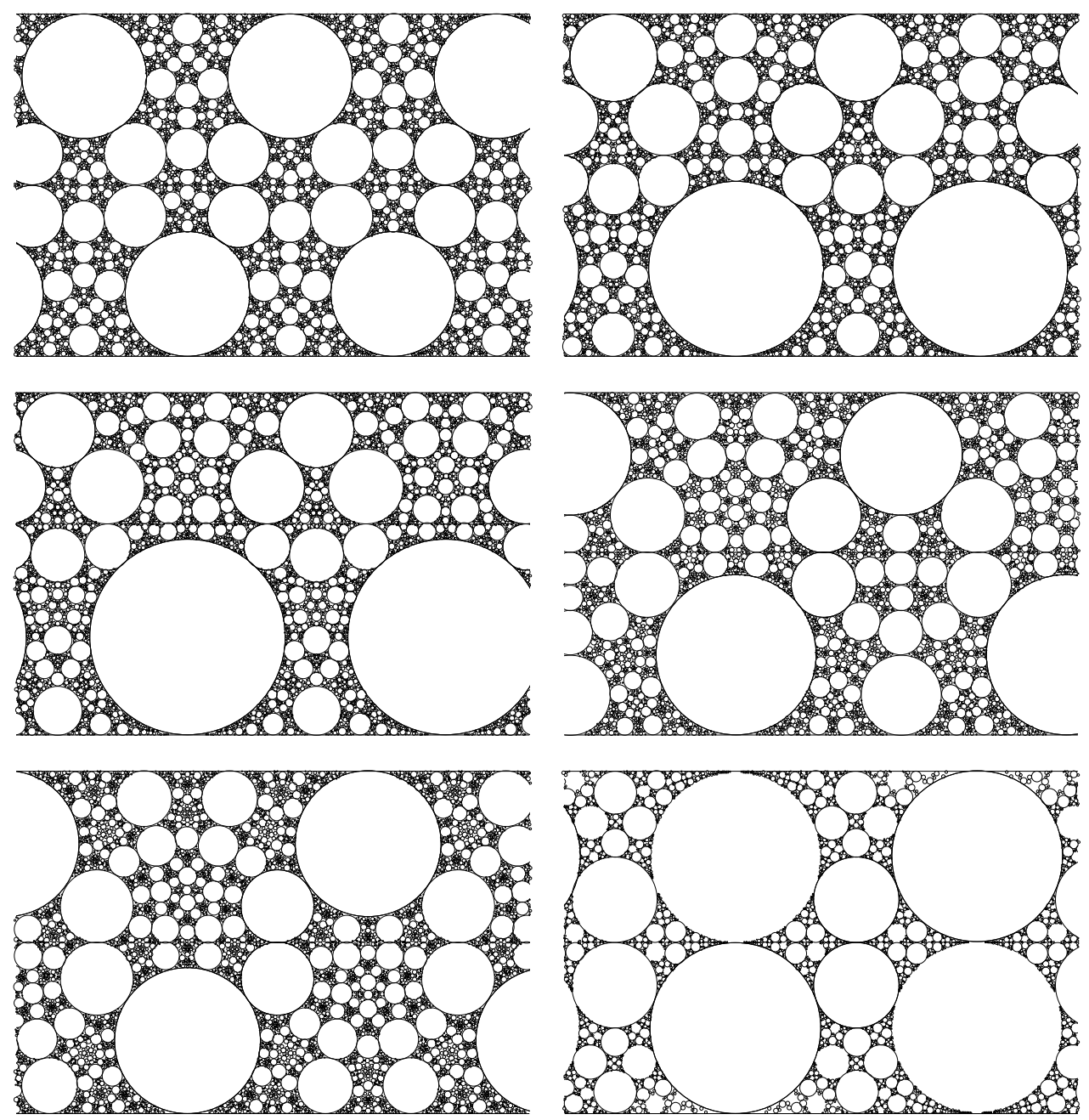

Figure 8. Some examples of F1 SFBs, for $l=6$ and for different $\left(n_{1}, n_{2}\right)$. Ordered from left to right and from top to bottom, in the format $\left(n_{1}, n_{2}\right):(0,0),(0,1),(0,2),(1,2),(2,2)$ and $(\infty, \infty)$.

\section{Discussion and conclusions}

In this paper we have shown the existence and studied the properties of SFBs with a basic loop having an arbitrary number of discs. We conclude that, as for the fourfold case, two topologically distinct families exist, the first having its consecutive copies of the seed touching each other and the second not, each one depending on two integer parameters $\left(n_{1}, n_{2}\right)$. We adapt the technique used previously in [1] to arbitrary loop sizes and formulate the equations that need to be fulfilled for the set of circles to be space filling. For low BLS $l$, we can analytically prove the existence and uniqueness of the solution. For larger $l$ we can only conclude that this was always the case observed numerically for any parameters $l, n_{1}$ and $n_{2}$ we have tried, and we believe that this is a general feature. This method permitted us to construct SFBs for various loop sizes and to study their properties, in particular their fractal dimension, which we have shown to be within a certain range of values (from 1.306 to 1.802) and which depend 


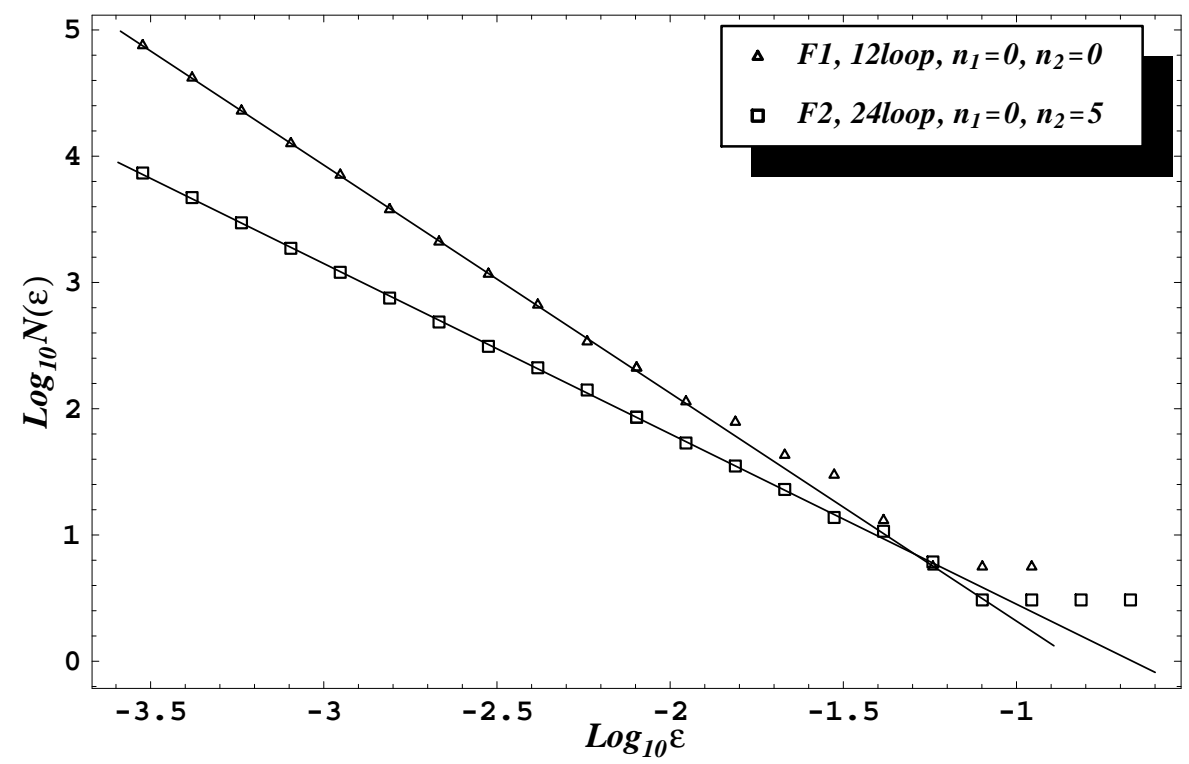

Figure 9. An example of the cumulative size distribution $N(\varepsilon)$ for two different realizations of SFBs. In the F1, 12, $(0,0) d_{f}=1.803$ and in the F2, 24, $(0,5) d_{f}=1.355$.

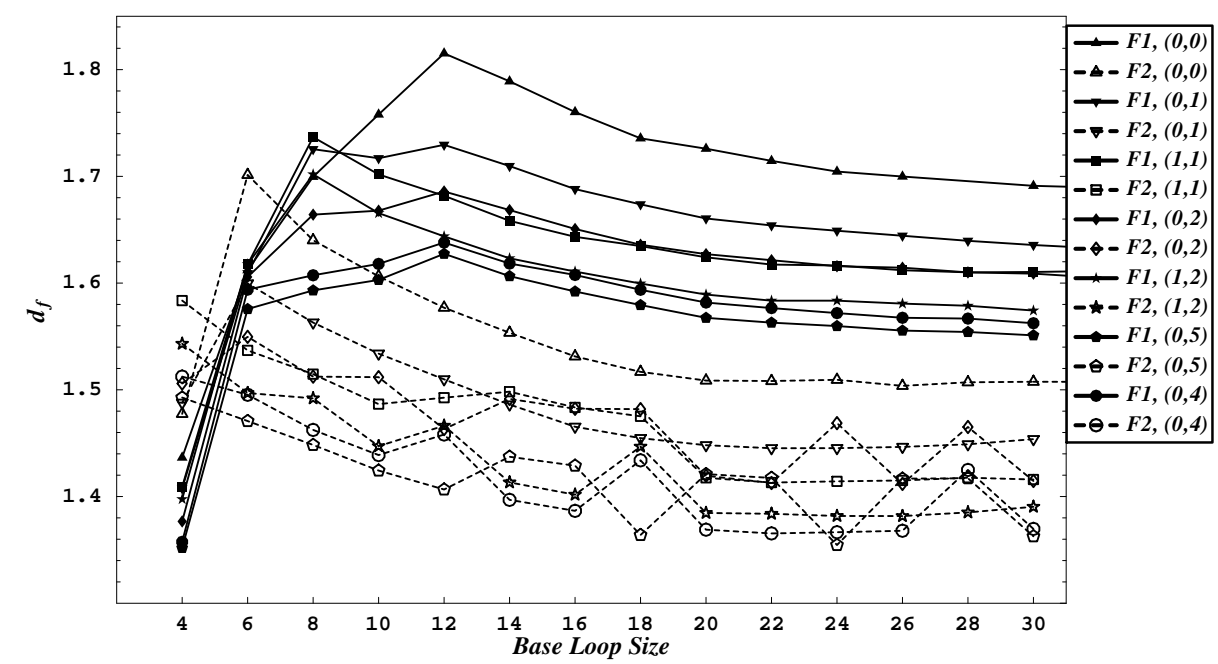

Figure 10. Variation of the fractal dimension $d_{f}$ as a function of the BLS for the two families (F1-full curves and full symbols, F2 - dashed curves and open symbols) for different $\left(n_{1}, n_{2}\right)$. The determination error of $d_{f}$ is of the order of the symbol size.

strongly on the chosen family, and on $l$.

This construction might be used as an idealized model for gauge materials or dense granulates in flows or in shear bands, and the extension we give here for larger BLS might give a more complete modelling than the four-element BLS given in [1]. In fact, it appears from figures 6 and 7 that the larger the BLS the greater the dominance of the small-sized circles, 


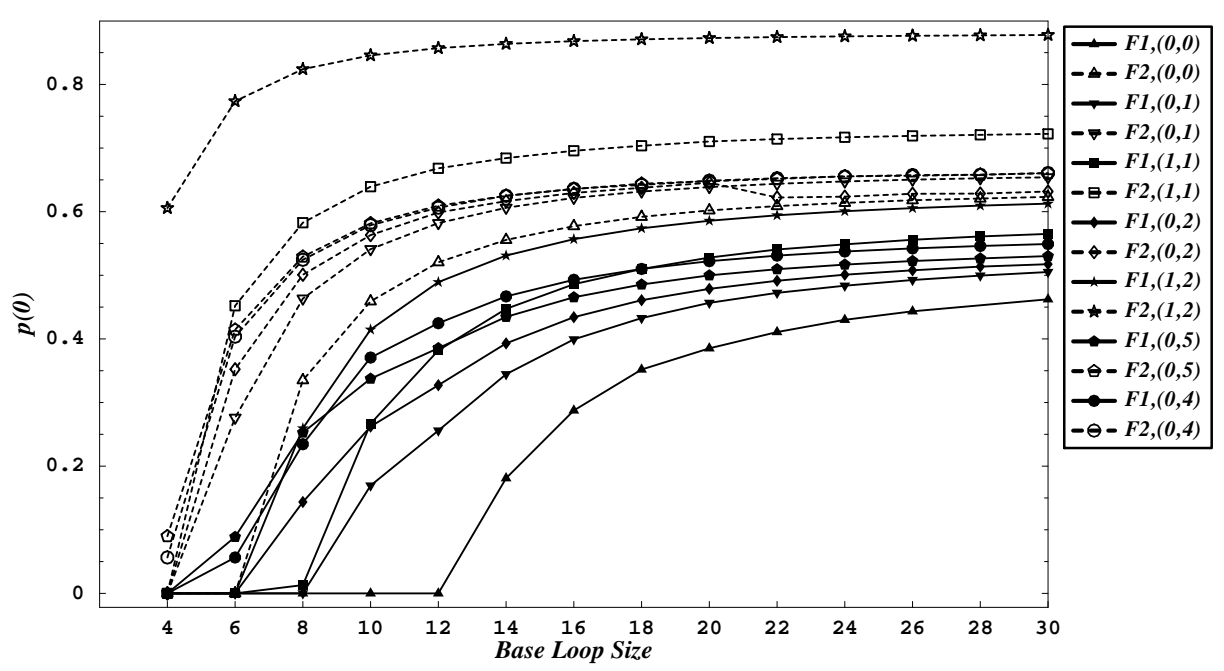

Figure 11. Variation of the fraction of uncovered area $p(0)$ as a function of the BLS for the two families (F1-full curves and full symbols, F2 - dashed curves and open symbols) for different $\left(n_{1}, n_{2}\right)$. The determination error of $p(0)$ is of the order of the symbol size.

which fits more closely to the observations of real gauge materials, naturally (e.g. sampled from the Lopez Canyon fault in the San Gabriel Mountains in Southern California, [9, 13]) or experimentally found in shear bands produced in a shear cell, [10,11].

In fact, in the references just quoted, when quartz sand with $d_{f}=0.8$ (in 2D projection) is subject to cyclic shear its size distribution changes, and its fractal dimension can reach about 1.7 when high shear rates are applied. In the real fault gauge, [9] reports values from 1.41 to 1.74. With the extension to arbitrary loop sizes this entire range can be covered by the SFB construction. We believe that the gauge under shear is pulverized and reorganizes itself into a form close to a SFB in order to minimize its energy loss due to friction.

A close look at the cumulative size-distribution figures shows that the upper limit for the power law distribution of sizes is of the order of 0.008 of the size of the strip; in fact, this upper cutoff was used in all of our calculations of the fractal dimension as the limit of applicability of (7). In [13] for the size distribution of a real fault gauge, the upper fractal limit observed was about $1 \mathrm{~cm}$. If the width of the fault is taken to be about $1 \mathrm{~m}$ the SFBs reproduce this property in agreement with the measured data.

In the future it would be interesting to investigate the possibility of building 3D SFBs. However, since the method used here depends on the properties of the complex plane, the 3D generalization might presently be hard to achieve.

\section{Appendix A}

\section{A.1. Properties of $z$}

In this section we will prove the following proposition.

Proposition 1. $1<z_{n} \equiv \cos ^{-2}[\pi /(n+3)] \leqslant 4, \forall n \in \mathbb{N}$.

In order to prove this property, we will first study the function $z(x)=\cos ^{-2}[\pi /(x+3)]$, for $x \in\left[0, \infty\left[\right.\right.$. Since $z^{\prime}(x)=-2 \pi \sec ^{2}(\pi /(x+3)) \tan (\pi /(x+3))(x+3)^{-2}<0$ in this range, 
$z(x)$ is strictly decreasing, and since $z(0)=4, \lim _{x \rightarrow \infty} z(x)=1$ and $z(x)$ is continuous, we conclude that $1<z(x) \leqslant 4$. This inequality still holds for $\mathbb{N}$ which is a subset of $[0, \infty[$, hence the property.

\section{A.2. Analytical expressions of the solutions for F1 and lesser BLSs}

In this section we give the analytical solution for the equation system (4) for BLSs up to eight. For any $l$, the solution was obtained by eliminating in equation system (4) the variables $r_{1}, r_{2}, a$ and $d_{1}, \ldots, d_{l-3}$, obtaining a polynomial equation in $d_{l-2}$. This equation was then solved either analytically, for small $l$, as we will describe in this section, or numerically for higher $l$. We will show in this section the existence and uniqueness of the solutions for $l$ up to eight and for all $n_{1}$ and $n_{2}$. We have numerically observed that this is also the case for higher $l$, but were unable to prove it.

A.2.1. F1-four-loop case. As mentioned previously, the case of a four-element BLS strip was treated in [1]. However, the method used was somewhat different from the one used here. The circles in seed were described by their radii rather than their contact points. This method was inadequate in the case of higher BLS since the invariance under the conformal transformations are complicated when the strip is represented in this manner.

In this case the polynomial equation for $d_{2}$ is

$$
-z_{n_{1}}-z_{n_{2}}+\left(z_{n_{1}}+z_{n_{2}}-1\right) d_{2}^{2}=0 .
$$

This equation has two real roots, $d_{2}= \pm\left(1-1 /\left(z_{n_{1}}+z_{n_{2}}\right)\right)^{-1 / 2}$, of which only the positive one is to be considered. When introduced into $(4 a)$ and $(4 b)$ the expressions for $d_{1}, r_{1}$ and $r_{2}$ are easily obtained, i.e.

$$
\begin{aligned}
& d_{1}=z_{n_{1}} /\left(\sqrt{\left(z_{n_{1}}+z_{n_{2}}\right)\left(z_{n_{1}}+z_{n_{2}}-1\right)}\right) \\
& r_{1,2}^{2}=z_{n_{1,2}} /\left(z_{n_{1}}+z_{n_{2}}-1\right) .
\end{aligned}
$$

Using $(4 c)$ and $(4 d)$ one can also retrieve the radii of the seed circles which are identical to the ones found in $[1,2]$.

A.2.2. F1-six-loop case. In the six-loop case the polynomial equation for $d_{4}$ is given by

$$
\begin{gathered}
z_{n_{1}}^{2}+z_{n_{1}}^{2}+z_{n_{1}} z_{n_{2}}+\left(2 z_{n_{1}}-2 z_{n_{1}}^{2}+2 z_{n_{2}}-2 z_{n_{1}} z_{n_{2}}-2 z_{n_{2}}^{2}\right) d_{4}^{2}+\left(1-2 z_{n_{1}}+z_{n_{1}}^{2}-2 z_{n_{2}}\right. \\
\left.+z_{n_{1}} z_{n_{2}}+z_{n_{2}}^{2}\right) d_{4}^{4}=0
\end{gathered}
$$

which is a quadratic equation in $d_{4}^{2}$ having the discriminant $2 z_{n_{1}} z_{n_{2}}$, hence having two real solutions for $d_{4}^{2}$. A trivial study of this equation, using the fact that $z_{n_{1}}>1$ and $z_{n_{2}}>1$, proves that both roots are positive, so that (A.4) has four different roots with exactly two of them positive. From the two solutions for $d_{4}$,

$$
d_{4_{ \pm}}=\left(\frac{z_{n_{1}}+z_{n_{2}} \pm \sqrt{z_{n_{1}} z_{n_{2}}}}{z_{n_{1}}+z_{n_{2}}-1 \pm \sqrt{z_{n_{1}} z_{n_{2}}}}\right)^{1 / 2}
$$

only $d_{4_{+}}$is acceptable since, from $(4 d)$ and $(4 c)$, we have $r_{1}^{2}=\left(d_{4}^{2}-1\right) z_{n_{1}}$ and it is easy to check that for $d_{4}$ we always get $r_{1}>1$, which is inadequate since the inversion circle will cross the line $\Gamma_{4}$ and hence the circle $\Gamma_{1}$ will have a diameter greater than the band width.

The distances $a, d_{1}, d_{2}, d_{3}$ and the inversion radii are deduced straightforwardly from $d_{4}$ using (4a) and (4b). 
A.2.3. F1-eight-loop case. The polynomial equation obtained for $d_{6}$ is

$z_{n_{1}}^{2}+z_{n_{2}}^{2}+d_{6}^{2}\left(2 z_{n_{1}}-2 z_{n_{1}}^{2}+2 z_{n_{2}}-2 z_{n_{2}}^{2}\right)+d_{6}^{4}\left(1-2 z_{n_{1}}+z_{n_{1}}^{2}-2 z_{n_{2}}+z_{n_{2}}^{2}\right)=0$

which is a quadratic equation in $d_{6}^{2}$ with a discriminant $8 z_{n_{1}} z_{n_{2}}$, thus having two real solutions in $d_{6}^{2}$ :

$$
d_{6_{ \pm}}^{2}=\frac{z_{n_{1}}^{2}+z_{n_{2}}^{2}-z_{n_{1}}-z_{n_{2}} \pm \sqrt{2 z_{n_{1}} z_{n_{2}}}}{z_{n_{1}}^{2}+z_{n_{2}}^{2}-2 z_{n_{1}}-2 z_{n_{2}}+1} .
$$

A short numerical study of $r_{1}^{2}=\left(d_{6}^{2}-1\right) z_{n_{1}}$ shows that only $d_{6_{-}}$has the adequate properties explained in appendix A.2.2. All the other unknowns of the equation system are deduced from $d_{6}$ using (4).

\section{A.3. Analytical expressions of the solutions for F2 and lesser BLSs}

In this section we give the analytical solution for the equation systems (5) for BLS up to eight. For larger BLS a numerical solution was found. In all cases a unique all-positive solution was found. For the F2 case, it is more useful to arrive at a polynomial equation involving $a$ rather than $d_{l-1}=d_{2 p}$.

A.3.1. F2-four-loop case. For a four-loop F2 case the equation is

$$
a^{2}=\left(z_{n_{1}}+z_{n_{2}}\right)^{-1}
$$

from which the other parameters are easily retrieved using (5). The results are the same as the one obtained in $[1,2]$.

A.3.2. F2-six-loop case. The polynomial equation for $a$ in this case is

$$
\left(z_{n_{1}}^{2}+z_{n_{2}}^{2}+z_{n_{1}} z_{n_{2}}\right) a^{4}-2\left(z_{n_{1}}+z_{n_{2}}\right) a^{2}+1=0 .
$$

Again, this equation is a quadratic one with regard to $a^{2}$, having $4 z_{n_{1}} z_{n_{2}}$ as its discriminant, thus having two real roots for $a^{2}$ of which only one is positive; hence only one solution is possible for $a$ :

$$
a=\left(z_{n_{1}}+z_{n_{2}}+\sqrt{z_{n_{1}} z_{n_{2}}}\right)^{-1 / 2} .
$$

All the other unknowns $\left(d_{1}, d_{2}, d_{3}\right.$ and $\left.d_{4}\right)$ are obtained straightforwardly from (5).

A.3.3. F2-eight-loop case. In this case the polynomial equation obtained after eliminating the variables $d_{i}$ is

$$
\left(z_{n_{1}}^{2}+z_{n_{2}}^{2}\right) a^{4}-2\left(z_{n_{1}}+z_{n_{2}}\right) a^{2}+1=0 .
$$

Since the discriminant is $8 z_{n_{1}} z_{n_{2}}>0$, there are two real roots for $a^{2}$, which are

$$
a_{ \pm}^{2}=\frac{z_{n_{1}}+z_{n_{2}} \pm \sqrt{2 z_{n_{1}} z_{n_{2}}}}{z_{n_{1}}^{2}+z_{n_{2}}^{2}} .
$$

We discard $a_{+}$since if $a=a_{+}$then from appendix A.1 $r_{1}^{2}+r_{2}^{2}=(x+y)(x+y+\sqrt{2 x y}) /$ $\left(x^{2}+y^{2}\right) \geqslant 2+\sqrt{2}$ and at least one of the inversion radii is greater than 1 , which is impossible. So, in this case also, one can have only one possible solution derived from $a=a_{-}$and (5).

Analytical solutions for higher BLS (up to 12-loop) do exist for F2, but these are too complicated to present here, and we were unable to analytically prove the uniqueness of the solution. 


\section{References}

[1] Herrmann H J, Mantica G and Bessis D 1990 Space-filling bearings Phys. Rev. Lett. 65 3223-6

[2] Herrmann H J 1990 Space-filling bearings Correlations and Connectivity ed H E Stanley and N Ostrowsky pp 108-20 (Dordrecht: Kluwer)

[3] McCann W, Nishenko S, Sykes L and Krause J 1979 Seismic gaps and plate tectonics: seismic potential for major boundaries Pure Appl. Geophys. 117 1082-147

[4] Lomnitz C 1982 What is a gap? Bull. Seismol. Soc. Am. 72 1411-13

[5] Boyd D W 1973 The residual set dimension of Apollonian packing Mathematika 20 170-4

[6] Thomas P B and Dhar D 1994 The Hausdorff dimension of the Apollonian packing of circles J. Phys. A: Math Gen. 27 2257-68

[7] Manna S S and Herrmann H J 1991 Precise determination of the fractal dimension of Apollonian packing and space-filling bearings J. Phys. A: Math. Gen. 24 L481-90

[8] Falconer K 1990 Fractal Geometry, Mathematical Foundations and Applications (New York: Wiley)

[9] Sammis C G, King G and Biegel R L 1987 The kinematics of gauge deformation Pure Appl. Geophys. 125 $777-812$

[10] Biegel R L and Sammis C G 1989 The frictional properties of a simulated gauge having a fractal particle distribution J. Struct. Geol. $11827-46$

[11] Marone C and Scholz C 1989 Particle size and microstructures in fault gauges J. Struct. Geol. 11 799-814

[12] Roux S, Herrmann H J, Hansen A and Vilotte J-P 1993 Model of gauge deformation: application to remanent magnetization Geophys. Res. Lett. 20 1499-502

[13] Sammis C G and Biegel R L 1989 Fractals, fault-gauge, and friction Pure Appl. Geophys. 131 255-71

[14] Oron G 1999 Empilements granulaires: calcul exact des contraintes et géométrie du remplissage PhD Thesis Université Paris 7-Denis Diderot (in French)

[15] Lavrentiev M and Chabat B 1977 Méthodes de la Théorie des Fonctions d'une Variable Complexe (Moscow: Mir) ch 2, pp 104-99

[16] Maxwell G 1982 Sphere packings and hyperbolic reflection groups J. Algebra $7978-97$

[17] Wolfram S 1996 The Mathematica Book (Cambridge: Cambridge University Press) 3rd edn

[18] Mandelbrot B B 1982 The Fractal Geometry of Nature (San Francisco, CA: Freeman)

[19] Wilker J B 1977 Sizing up a solid packing Period. Math. Hung. 8 117-34

[20] Eggleston H G 1957 Problems in Euclidean space: application of convexity (International Series of Monographs in Pure and Applied Mathematics) (Oxford: Pergamon) 